\title{
Exact Solutions of Rayleigh-Stokes Problem for Heated Generalized Maxwell Fluid in a Porous Half-Space
}

\author{
Changfeng $\mathrm{Xue}^{\mathbf{1}}$ and Junxiang $\mathrm{Ni}^{2}$ \\ ${ }^{1}$ Department of Fundamental Sciences, Yancheng Institute of Technology, Yancheng, \\ Jiangsu 224003, China \\ ${ }^{2}$ Department of Mathematics, School of Science, Beijing Jiaotong University, Beijing 100044, China
}

Correspondence should be addressed to Changfeng Xue, chfxue@gmail.com

Received 8 October 2007; Accepted 24 April 2008

Recommended by Katica Hedrih

The Rayleigh-Stokes problem for a generalized Maxwell fluid in a porous half-space with a heated flat plate is investigated. For the description of such a viscoelastic fluid, a fractional calculus approach in the constitutive relationship model is used. By using the Fourier sine transform and the fractional Laplace transform, exact solutions of the velocity and the temperature are obtained. Some classical results can be regarded as particular cases of our results, such as the classical solutions of the first problem of Stokes for Newtonian viscous fluids, Maxwell fluids, and Maxwell fluids in a porous half-space.

Copyright ( 2008 C. Xue and J. Nie. This is an open access article distributed under the Creative Commons Attribution License, which permits unrestricted use, distribution, and reproduction in any medium, provided the original work is properly cited.

\section{Introduction}

Many materials such as clay coatings, drilling mud, suspensions, certain oils and greases, polymer melts, elastomers, and many emulsions have been treated as non-Newtonian fluids. It is difficult to suggest a single model which can exhibit all properties of non-Newtonian fluids as is done for Newtonian fluids. They cannot be described in a simple model as for the Newtonian fluids, and there has been much confusion over the classification of the non-Newtonian fluids. For this reason, many models of constitutive equations have been proposed. Recently, fractional calculus has encountered much success in the description of constitutive relations of viscoelastic fluids. The starting point of the fractional derivative model of viscoelastic fluids is usually a classical differential equation which is modified by replacing the time derivative of an integer order with the so-called Riemann-Liouville fractional calculus operator. This generalization allows one to define precisely noninteger order integrals or 
derivatives. Bagley and Torvik [1], Friedrich [2], Huang et al. [3], He et al. [4], Xu and Tan [5-7], Tan [8-12], and Shen et al. [13,14] have sequentially introduced the fractional calculus approach into various rheology problems. Fractional derivatives have been found to be quite flexible in describing viscoelastic behaviors.

The first problem of Stokes for the flat plate, like the Rayleigh-Stokes problem for an edge, has received much attention because of its practical importance [15-18]. This unsteady flow problem examines the diffusion of vorticity in a half-space filled with a viscous incompressible fluid that is set to motion when an infinite flat plate suddenly assumes a constant velocity parallel to itself from the rest. By using similarity transformation of variables, the exact solution corresponding to a Newtonian fluid was obtained in an elegant form by Stokes. But for a Maxwell fluid, it is difficult to obtain such a strict similarity solution. Further, the order of the equation of motion for such a fluid is higher than that of the NavierStokes equation and thus, in general, one needs conditions in addition to the usual adherence boundary condition. Friedrich first investigated this problem and gave a few exact solutions [19].

On the other hand, the problems of the fluid through porous media play an important role in various practical applications. But very little efforts have so far been made to discuss these problems. Recently, based on the local volume averaging technique and the balance of forces acting on a volume element of viscoelastic fluids in porous media, Tan and Masuoka [20-23] developed a modified Darcy-Brinkman model for viscoelastic fluid flows in porous media. Based on their works, in this paper, we extend Stokes' first problem to that of a heated generalized Maxwell fluid with fractional derivative in a porous half-space. Exact solutions of the velocity and the temperature are obtained by using the Fourier sine transform and the fractional Laplace transform. Some previous classical results can be recovered from our results, such as the classical solutions of the first problem of Stokes for Newtonian viscous fluids and those for Maxwell fluids.

\section{Basic equations}

The well-known Darcy law states that, in the flow of a Newtonian fluid through a porous medium, the pressure gradient $\nabla p$ caused by the friction drag is directly proportional to the velocity, namely,

$$
\nabla p=-\frac{\mu}{K} V_{D}
$$

where $V_{D}$ is the Darcian velocity, $\mu$ is the dynamic viscosity of the fluid, and $K$ is the permeability of the porous medium, respectively. The Darcy law, however, does not consider the boundary effect on the flow, which unfortunately cannot be ignored in many applications. In 1947, Brinkman proposed an equation to describe the locally averaged flow in a porous medium [24]. In the Brinkman equation, the pressure gradient, the divergence of the viscous stress tensor, and the friction force exerted by the porous medium satisfy

$$
-\nabla p+\mu_{e} \nabla^{2} V_{D}-\frac{\mu}{K} V_{D}=0,
$$

where $\mu_{e}$ is the effective dynamic viscosity of the medium. Although the Brinkman equation takes the boundary effect on the flow into account, it is justified for and can thus be applied to 
only steady-state flows of a Newtonian fluid in porous media. For viscoelastic fluid flows in porous media, on the other hand, only a few mathematical macroscopic filtration models have been proposed. In particular, by analogy with the constitutive equation of a Maxwell fluid $[25,26]$, the following phenomenological model has been introduced:

$$
\left(1+t_{m}^{\alpha} \frac{\partial^{\alpha}}{\partial t^{\alpha}}\right) \nabla p=-\frac{\mu}{K} V_{D}
$$

where $t_{m}$ is the relaxation time of the generalized Maxwell fluid, $\alpha(0<\alpha \leq 1)$ is fractional coefficient, and $\partial^{\alpha} \tau / \partial t^{\alpha}=D_{t}^{\alpha}$ is Riemann-Liouville fractional derivative, which is defined as in [27]

$$
{ }_{a} D_{t}^{\alpha}[f(t)]=\frac{1}{\Gamma(k-\alpha)} \frac{d^{k}}{d t^{k}} \int_{a}^{t}(t-\tau)^{k-\alpha-1} f(\tau) d \tau \quad(k-1 \leq \alpha<k),
$$

where $\Gamma(\cdot)$ is the gamma function.

Let us now follow the same procedure as used in [22] to investigate the flow of a generalized Maxwell fluid in a porous half-space. With the positive $y$-axis of the rectangular coordinate system pointing up, a generalized Maxwell fluid flows through the porous halfspace $(y>0)$ above and in contact with a flat plate occupying the $x z$-plane. At time $t=0$, both the fluid and the plate are at rest. At time $t=0^{+}$, the flat plate suddenly starts to slide in its plane with a constant speed $U_{0}$. Under these conditions, no flow occurs in the $y$ - and the $z$-directions, and the flow velocity at a given point in the porous half-space depends only on its $y$-coordinate and time $t$, that is, the intrinsic velocity field takes the form

$$
V=u(y, t) i
$$

where $i$ represents the unit vector in the $x$-direction. The intrinsic velocity $V$, averaged over the pore space, is related to the Darcian velocity by $V_{D}=\phi V$, where $\phi$ is the porosity of the porous medium. In this work, employing a local volume averaging technique [20, 28-30], we will consider a modified, more rigorous Darcy law for Stokes' first problem with a generalized Maxwell fluid in the porous half-space. In fact, in terms of the pressure gradient, the local volume-averaged balance of linear momentum can be given by

$$
\rho \frac{\partial V}{\partial t}=-\nabla p+\nabla \tau+r
$$

where $\rho$ is the fluid density, $\tau$ is the viscous stress tensor, and $r$ is the Darcy resistance, namely, the viscous damping force caused by the micropore structure of a porous medium, respectively. Under the assumption that

$$
\tau+t_{m}^{\alpha}\left[\frac{\partial^{\alpha} \tau}{\partial t^{\alpha}}+(V \cdot \nabla) \tau-(\nabla V)^{T} \tau-\tau(\nabla V)\right]=\mu\left[\nabla V+(\nabla V)^{T}\right],
$$

as in $[23,31]$, next we discuss the viscoelastic properties of the fluid using the upper convected derivative generalized Maxwell fluid. 
It should be pointed out that the classical viscous Newtonian fluid is just a special case of this model at $t_{m}=0$. Inserting (2.5) into (2.7) and using the initial condition $\tau(y, 0)=0$, we get $\tau_{x z}=\tau_{y y}=\tau_{y z}=\tau_{z z}=0$ and

$$
\begin{gathered}
\tau_{x x}+t_{m}^{\alpha} \frac{\partial^{\alpha} \tau_{x x}}{\partial t^{\alpha}}=2 \tau_{x y} \frac{\partial u}{\partial y}, \\
\tau_{x y}+t_{m}^{\alpha} \frac{\partial^{\alpha} \tau_{x y}}{\partial t^{\alpha}}=\mu \frac{\partial u}{\partial y} .
\end{gathered}
$$

Note that the pressure gradient $\nabla p$ given in (2.3) can be taken as a measure of the same resistance to flow in the bulk of the porous medium [26]. Since the Darcy resistance $r$ in (2.6) is also in effect a measure of the flow resistance offered by the solid matrix, from (2.3) we get

$$
\left(1+t_{m}^{\alpha} \frac{\partial^{\alpha}}{\partial t^{\alpha}}\right) r=-\frac{\mu}{K} V_{D}
$$

Using (2.9) and (2.10) to eliminate $\tau$ and $r$ in (2.6) and recalling that $V_{D}=\phi V$, we have the momentum equation of Stokes' first problem for a generalized Maxwell fluid in the porous half-space

$$
\rho\left(1+t_{m}^{\alpha} \frac{\partial^{\alpha}}{\partial t^{\alpha}}\right) \frac{\partial u}{\partial t}=-\left(1+t_{m}^{\alpha} \frac{\partial^{\alpha}}{\partial t^{\alpha}}\right) \frac{\partial p}{\partial x}+\mu \frac{\partial^{2} u}{\partial y^{2}}-\frac{\mu \phi}{K} u
$$
as follows:

Ignoring the pressure gradient in the $x$-direction finally leads to the equation of motion

$$
\rho\left(1+t_{m}^{\alpha} \frac{\partial^{\alpha}}{\partial t^{\alpha}}\right) \frac{\partial u}{\partial t}=\mu \frac{\partial^{2} u}{\partial y^{2}}-\frac{\mu \phi}{K} u .
$$

In addition, the initial and the boundary conditions are

$$
\begin{gathered}
u(y, 0)=0, \\
u(0, t)=U_{0}
\end{gathered}
$$

respectively, and the natural condition is

$$
u(y, t) \longrightarrow 0, \quad \frac{\partial u(y, t)}{\partial y} \longrightarrow 0 \quad \text { as } y \longrightarrow \infty .
$$

We will assume that there is a local thermal equilibrium. Then, $T_{s}=T_{f}=T$, where $T_{s}$ and $T_{f}$ are the temperatures of the solid and the fluid phases in porous media, respectively. The thermal energy equation is then $[32,33]$

$$
(\rho c)_{f s} \frac{\partial T}{\partial t}=\lambda_{e} \frac{\partial^{2} T}{\partial y^{2}}+Q_{T}
$$

where $(\rho c)_{f s}$ is the specific heat capacity of the system formed by both the fluid and the solid phases. More precisely, $(\rho c)_{f s}=(1-\phi) \rho_{s} c_{s}+\phi c_{f} \rho$, where $\rho_{s}$ is the density of the solid phase, $c_{S}$ is the specific heat capacity of the solid phase, and $c_{f}$ is the specific heat capacity of the fluid 
at a constant pressure, respectively. $\lambda_{e}$ is the equivalent thermal conductivity of the fluid in the porous half-space, and $Q_{T}$ is the contribution due to viscous dissipation [34, 35]

$$
Q_{T}=\frac{\mu \phi^{2}}{K} u^{2}+\mu\left(\frac{\partial u}{\partial y}\right)^{2}
$$

Equation (2.16) is a unified form compatible with an expression derived from the NavierStokes equation for a fluid clear of solid [36, 37].

The corresponding initial and boundary conditions are

$$
\begin{gathered}
T(y, 0)=T_{1}, \\
T(0, t)=T_{0}(t) .
\end{gathered}
$$

Moreover, the natural conditions are

$$
T(y, t) \longrightarrow T_{1}, \quad \frac{\partial T(y, t)}{\partial y} \longrightarrow 0 \quad \text { as } y \longrightarrow \infty
$$

\section{Exact solutions}

Employing the nondimensional quantities

$$
u^{*}=\frac{u}{U_{0}}, \quad y^{*}=\frac{y U_{0}}{v}, \quad t^{*}=\frac{t U_{0}^{2}}{v}, \quad T^{*}=\frac{T-T_{1}}{T_{0}(0)-T_{1}},
$$

the dimensionless motion and energy equations can be written as (for brevity the dimensionless marks " $*$ " are omitted here)

$$
\begin{gathered}
\left(1+\gamma \frac{\partial^{\alpha}}{\partial t^{\alpha}}\right) \frac{\partial u}{\partial t}=\frac{\partial^{2} u}{\partial y^{2}}-\beta^{2} u, \\
u(y, 0)=0, \\
u(0, t)=1, \\
u(y, t) \longrightarrow 0, \quad \frac{\partial u(y, t)}{\partial y} \longrightarrow 0 \quad \text { as } y \longrightarrow \infty, \\
\frac{\partial T}{\partial t}=\xi \frac{\partial^{2} T}{\partial y^{2}}+Q_{t}(y, t), \\
T(y, 0)=0, \\
T(0, t)=f(t), \\
\frac{\partial T(y, t)}{\partial y} \longrightarrow 0 \quad \text { as } y \longrightarrow \infty .
\end{gathered}
$$

where $\gamma=\left(t_{m} U_{0}^{2} / v\right)^{\alpha}, \beta=v /\left(U_{0} \sqrt{K / \phi}\right), \xi=D_{e} / v, f(t)=\left(T_{0}(t)-T_{1}\right) /\left(T_{0}(0)-T_{1}\right), D_{e}=$ $\lambda_{e} /(\rho c)_{f s} \cdot v=\mu / \rho$ is the kinematic viscosity. The dimensionless viscous dissipation, namely, $Q_{t}(y, t)$, has the following form:

$$
Q_{t}(y, t)=\frac{\phi^{2} \mu^{2}}{(\rho c)_{f s} K\left(T_{0}(0)-T_{1}\right) \rho} u^{2}+\frac{U_{0}^{2} \rho}{(\rho c)_{f s}\left(T_{0}(0)-T_{1}\right)}\left(\frac{\partial u}{\partial y}\right)^{2} .
$$




\subsection{Solution of the velocity field}

Multiplying both sides of (3.2) and (3.3) by integrating with respect to $y$ from 0 to $\infty$, and then using the boundary conditions of (3.4) and (3.5), in fact we have

$$
\begin{aligned}
\frac{\partial U(\zeta, t)}{\partial t}+\gamma \frac{\partial^{\alpha} U(\zeta, t)}{\partial t^{\alpha}} & =-\zeta^{2} U(\zeta, t)+\zeta-\beta^{2} U(\zeta, t), \\
U(\zeta, 0) & =0
\end{aligned}
$$

where $U(\zeta, t)$ denotes the Fourier sine transform of $U(y, t)$ with respect to $y$. In order to obtain an exact solution of (3.11) subject to the initial condition of (3.12), the fractional Laplace transform is used. Let

$$
\bar{U}(\zeta, s)=L\{U(\zeta, t)\}=\int_{0}^{\infty} e^{-s t} U(\zeta, t) d t
$$

be the image function of $U(\zeta, t)$, where $s$ is the transform parameter. Using Laplace transform principle of sequential fractional derivatives [27], we get

$$
\bar{U}(\zeta, s)=\frac{\zeta}{s\left[\gamma s^{\alpha+1}+s+\left(\zeta^{2}+\beta^{2}\right)\right]} .
$$

In order to avoid the burdensome calculations of residues and contour integrals, we apply the discrete inverse Laplace transform method here. First, we rewrite (3.14) in a series form

$$
\bar{U}(\zeta, s)=\frac{\zeta}{\zeta^{2}+\beta^{2}} \sum_{k=0}^{\infty}(-1)^{k}\left(\frac{\zeta^{2}+\beta^{2}}{\gamma}\right)^{k+1} \frac{s^{-k-2}}{\left(s^{\alpha}+\gamma^{-1}\right)^{k+1}} .
$$

Then, applying the inversion formulae term by term for the Laplace transform, (3.15) becomes

$$
U(\zeta, t)=\frac{\zeta}{\zeta^{2}+\beta^{2}} \sum_{k=0}^{\infty} \frac{(-1)^{k}}{k !}\left(\frac{\zeta^{2}+\beta^{2}}{\gamma}\right)^{k+1} t^{(k+1)(\alpha+1)} E_{\alpha, \alpha+k+2}^{(k)}\left(\frac{-t^{\alpha}}{\gamma}\right)
$$

in which

$$
E_{\alpha, \beta}(t)=\sum_{k=0}^{\infty} \frac{t^{k}}{\Gamma(\alpha k+\beta)}
$$

denotes the generalized Mittag-Leffler function. Here, we use the following property of the generalized Mittag-Leffler function's inverse Laplace transform [27]

$$
L^{-1}\left[\frac{n ! s^{\lambda-\mu}}{\left(s^{\lambda} \mp c\right)^{n+1}}\right]=t^{\lambda n+\mu-1} E_{\lambda, \mu}^{(n)}\left( \pm c t^{\lambda}\right) \quad\left(\operatorname{Re}(s)>|c|^{1 / \lambda}\right) .
$$


Inverting (3.18) by the Fourier sine inverse transform, we obtain an exact solution to the velocity field as follows:

$$
u(y, t)=\frac{2}{\pi} \int_{0}^{\infty} \frac{\zeta \sin (\zeta y)}{\zeta^{2}+\beta^{2}} \sum_{k=0}^{\infty} \frac{(-1)^{k}}{k !}\left(\frac{\zeta^{2}+\beta^{2}}{\gamma}\right)^{k+1} t^{(k+1)(\alpha+1)} E_{\alpha, \alpha+k+2}^{(k)}\left(\frac{-t^{\alpha}}{\gamma}\right) d \zeta .
$$

In a special case, when $\alpha=1$, corresponding to Stokes' first problem for a Maxwell fluid in a porous half-space, (3.14) can be written as

$$
\bar{U}(\zeta, s)=\frac{\zeta}{s\left[\gamma s^{2}+s+\left(\zeta^{2}+\beta^{2}\right)\right]}
$$

Correspondingly, (3.19) can be written as

$$
u(y, t)=\frac{2}{\pi} \int_{0}^{\infty} \frac{\zeta \sin (\zeta y)}{\zeta^{2}+\beta^{2}} \sum_{k=0}^{\infty} \frac{(-1)^{k}}{k !}\left(\frac{\zeta^{2}+\beta^{2}}{\gamma}\right)^{k+1} t^{2(k+1)} E_{1, k+3}^{(k)}\left(\frac{-t}{\gamma}\right) d \zeta,
$$

which is just the dimensionless velocity solution of a Maxwell fluid in a porous half-space obtained in another form by Tan [22].

In a special case, when $\alpha=1$ and $\beta=0$, we have

$$
u(y, t)=\frac{2}{\pi} \int_{0}^{\infty} \frac{\sin (\zeta y)}{\zeta} \sum_{k=0}^{\infty} \frac{(-1)^{k}}{k !}\left(\frac{\zeta^{2}}{\gamma}\right)^{k+1} t^{2(k+1)} E_{1, k+3}^{(k)}\left(\frac{-t}{\gamma}\right) d \zeta,
$$

which is just the dimensionless velocity solution of a clear Maxwell fluid obtained in another form by Jordan et al. [38].

In another special case, when $\alpha=1, \beta=0$, and $\gamma=0$, corresponding to the classical version of Stokes' first problem for a Newtonian fluid, (3.14) can be written as

$$
\bar{U}(\zeta, s)=\frac{\zeta}{s\left(s+\zeta^{2}\right)}
$$

Correspondingly, (3.19) can be written as

$$
u(y, t)=1-\frac{2}{\pi} \int_{0}^{\infty} \frac{\sin (\zeta y)}{\zeta} \exp \left(-\zeta^{2} t\right) d \zeta
$$

Equation (3.24) can be simplified further as follows:

$$
u(y, t)=1-\operatorname{erf}\left(\frac{y}{2 \sqrt{t}}\right)
$$

where $\operatorname{erf}(x)$ is the error function of Gauss. This is just the well-known result of Stokes' first problem for a clear Newtonian fluid. 


\subsection{Solution of temperature field}

In order to determine the temperature distribution in the porous half-space, we multiply both sides of (3.6) and (3.7) by $\sin (s y)$ and then integrate with respect to $y$ from 0 to $\infty$. Using (3.8) and (3.9), we get

$$
\begin{aligned}
\frac{\partial \bar{T}(s, t)}{\partial t}+\xi s^{2} \bar{T}(s, t) & =\xi s f(t)+g(s, t), \\
\bar{T}(s, 0) & =0
\end{aligned}
$$

where $\bar{T}(s, t)$ and $g(s, t)$ denote the Fourier sine transforms of $T(y, t)$ and $Q_{t}(y, t)$ with respect to $y$, respectively. Since the solution of velocity $u(y, t)$ is obtained by $(3.21), g(s, t)$ is a known function. The solution of the ordinary differential equation (3.26) subject to the initial condition (3.27) can be easily obtained as

$$
\bar{T}(s, t)=\exp \left(-\xi s^{2} t\right) \int_{0}^{t}(\xi s f(\tau)+g(s, \tau)) \exp \left(\xi s^{2} t\right) d \tau
$$

Inverting (3.28) by the Fourier sine inverse transform, and integrating by parts the term containing $f(\tau)$, we get

$$
\begin{aligned}
T(y, t)= & f(0)\left(1-\operatorname{erf}\left(\frac{y}{2 \sqrt{\xi t}}\right)\right)+\int_{0}^{t} f^{\prime}(\tau)\left(1-\operatorname{erf}\left(\frac{y}{2 \sqrt{\xi(t-\tau)}}\right)\right) d \tau \\
& +\frac{2}{\pi} \int_{0}^{\infty} \int_{0}^{t} g(s, t) \exp \left(-\xi s^{2}(t-\tau)\right) \sin (s y) d \tau d s .
\end{aligned}
$$

In a special case, when $U_{0}=0$, the fluid is at rest, and the temperature distribution is the same for both a Maxwell fluid and a Newtonian one. Then, (3.29) takes the form

$$
T(y, t)=f(0)\left(1-\operatorname{erf}\left(\frac{y}{2 \sqrt{\xi t}}\right)\right)+\int_{0}^{t} f^{\prime}(\tau)\left(1-\operatorname{erf}\left(\frac{y}{2 \sqrt{\xi(t-\tau)}}\right)\right) d \tau
$$

From (3.30), we easily obtain that

$$
T(y, t) \longrightarrow T_{0}(\infty) \text { as } t \longrightarrow \infty \text {. }
$$

If, moreover, the plate is kept at a constant temperature, (3.31) reduces to a well-known form,

$$
T(y, t)=f(0)\left(1-\operatorname{erf}\left(\frac{y}{2 \sqrt{\xi t}}\right)\right) .
$$

\section{Conclusion}

In this work, we have presented some results about the generalized Maxwell fluid in a porous half-space on a heated flat plate. An exact solution of the velocity field was obtained by using the Fourier sine transform and the fractional Laplace transform. The temperature distribution in the generalized Maxwell fluid in the porous half-space subject to a linear flow on a heated flat plate was determined. Some classical results can be considered as particular cases of our results, such as the solutions corresponding to a Newtonian viscous fluid, a clear Maxwell fluid, and a Maxwell fluid in a porous half-space. They can be easily obtained from the results presented in this paper by letting $\alpha=1, \beta=0, \gamma=0 ; \alpha=1, \beta=0$, and $\alpha=1$, respectively. It has also been shown that the fractional constitutive relationship model is more flexible than the conventional model in describing the properties of viscoelastic fluids. 


\section{Acknowledgments}

This work was supported by National Natural Science Foundation of China (Grants no. 10572006, 10632010, 40628001) and National Key Basic Research Program (2006CB705803).

\section{References}

[1] R. L. Bagley and P. J. Torvik, "A theoretical basis for the application of fractional calculus to viscoelasticity," Journal of Rheology, vol. 27, no. 3, pp. 201-210, 1983.

[2] C. Friedrich, "Relaxation and retardation functions of the Maxwell model with fractional derivatives," Rheologica Acta, vol. 30, no. 2, pp. 151-158, 1991.

[3] J. Huang, G. He, and C. Liu, "Analysis of general second-order fluid flow in double cylinder rheometer," Science in China Series A, vol. 40, no. 2, pp. 183-190, 1997.

[4] G. He, J. Huang, and C. Liu, "General second order fluid flow in a pipe," Applied Mathematics and Mechanics, vol. 16, no. 9, pp. 825-831, 1995.

[5] M.-Y. Xu and W.-C. Tan, "Theoretical analysis of the velocity field, stress field and vortex sheet of generalized second order fluid with fractional anomalous diffusion," Science in China Series A, vol. 44, no. 11, pp. 1387-1399, 2001.

[6] M.-Y. Xu and W.-C. Tan, "The representation of the constitutive equation of viscoelastic materials by the generalized fractional element networks and its generalized solutions," Science in China Series A, vol. 32, no. 8, pp. 673-681, 2002.

[7] M.-Y. Xu and W.-C. Tan, "Intermediate processes and critical phenomena: theory, method and progress of fractional operators and their applications to modern mechanics," Science in China Series G, vol. 49, no. 3, pp. 257-272, 2006.

[8] W.-C. Tan and M.-Y. Xu, "The impulsive motion of flat plate in a general second grade fluid," Mechanics Research Communication, vol. 29, no. 1, pp. 3-9, 2002.

[9] W.-C. Tan and M.-Y. Xu, "Plane surface suddenly set in motion in a viscoelastic fluid with fractional Maxwell model," Acta Mechanica Sinica, vol. 18, no. 4, pp. 342-349, 2002.

[10] W.-C. Tan, F. Xian, and L. Wei, "An exact solution of unsteady Couette flow of generalized second grade fluid," Chinese Science Bulletin, vol. 47, no. 21, pp. 1783-1785, 2002.

[11] W.-C. Tan, W. X. Pan, and M.-Y. Xu, "A note on unsteady flows of a viscoelastic fluid with the fractional Maxwell model between two parallel plates," International Journal of Non-Linear Mechanics, vol. 38, no. 5, pp. 645-650, 2003.

[12] W.-C. Tan and M.-Y. Xu, "Unsteady flows of a generalized second grade fluid with the fractional derivative model between two parallel plates," Acta Mechanica Sinica, vol. 20, no. 5, pp. 471-476, 2004.

[13] F. Shen, W.-C. Tan, Y.-H. Zhao, and T. Masuoka, "Decay of vortex velocity and diffusion of temperature in a generalized second grade fluid," Applied Mathematics and Mechanics, vol. 25, no. 10, pp. 1151-1159, 2004.

[14] F. Shen, W.-C. Tan, Y. Zhao, and T. Masuoka, "The Rayleigh-Stokes problem for a heated generalized second grade fluid with fractional derivative model," Nonlinear Analysis: Real World Applications, vol. 7, no. 5, pp. 1072-1080, 2006.

[15] I. Teipel, "The impulsive motion of a flat plate in a viscoelastic fluid," Acta Mechanica, vol. 39, no. 3-4, pp. 277-279, 1981.

[16] K. R. Rajagopal, "A note on unsteady unidirectional flows of a non-Newtonian fluid," International Journal of Non-Linear Mechanics, vol. 17, no. 5-6, pp. 369-373, 1982.

[17] K. R. Rajagopal and A. S. Gupta, "On a class of exact solutions to the equations of motion of a second grade fluid," International Journal of Engineering Science, vol. 19, no. 7, pp. 1009-1014, 1981.

[18] K. R. Rajagopal and A. S. Gupta, "An exact solution for the flow of a non-Newtonian fluid past an infinite porous plate," Meccanica, vol. 19, no. 2, pp. 158-160, 1984.

[19] C. Friedrich, "Relaxation and retardation functions of the Maxwell model with fractional derivatives," Rheologica Acta, vol. 30, no. 2, pp. 151-158, 1991.

[20] W.-C. Tan and T. Masuoka, "Stokes' first problem for a second grade fluid in a porous half-space with heated boundary," International Journal of Non-Linear Mechanics, vol. 40, no. 4, pp. 515-522, 2005.

[21] W.-C. Tan and T. Masuoka, "Stokes' first problem for an Oldroyd-B fluid in a porous half space," Physics of Fluids, vol. 17, no. 2, Article ID 023101, 7 pages, 2005. 
[22] W.-C. Tan, "Velocity overshoot of start-up flow for a Maxwell fluid in a porous half-space," Chinese Physics, vol. 15, no. 11, pp. 2644-2650, 2006.

[23] W.-C. Tan and T. Masuoka, "Stability analysis of a Maxwell fluid in a porous medium heated from below," Physics Letters A, vol. 360, no. 3, pp. 454-460, 2007.

[24] F. Capuani, D. Frenkel, and C. P. Lowe, "Velocity fluctuations and dispersion in a simple porous medium," Physical Review E, vol. 67, no. 52, Article ID 056306, 8 pages, 2003.

[25] G. M. Alisaev and A. K. Mirzadjanzade, "For the calculation of delay phenomenon in filtration theory," Izvestiya Vysshikh Uchebnykh Zavedeniy. Neft' $i$ Gaz, vol. 6, pp. 71-78, 1975.

[26] B. Khuzhayorov, J.-L. Auriault, and P. Royer, "Derivation of macroscopic filtration law for transient linear viscoelastic fluid flow in porous media," International Journal of Engineering Science, vol. 38, no. 5, pp. 487-504, 2000.

[27] I. Podlubny, Fractional Differential Equations, Mathematics in Science and Engineering, Academic Press, San Diego, Calif, USA, 1999.

[28] K. Vafai and C. L. Tien, "Boundary and inertia effects on flow and heat transfer in porous media," International Journal of Heat and Mass Transfer, vol. 24, no. 2, pp. 195-203, 1981.

[29] T. Masuoka and Y. Takatsu, "Turbulence characteristics in porous media," in Transport Phenomena in Porous Media Vol. II, D. B. Ingham and I. Pop, Eds., pp. 231-256, Pergamon Press, Oxford, UK, 2002.

[30] D. B. Ingham and I. Pop, Eds., Transport Phenomena in Porous Media II, Pergamon Press, Oxford, UK, 2002.

[31] P. N. Kaloni and J. X. Lou, "Stability of Hadley circulations in a Maxwell fluid," Journal of NonNewtonian Fluid Mechanics, vol. 103, no. 2-3, pp. 167-186, 2002.

[32] A. Younes, "On modelling the multidimensional coupled fluid flow and heat or mass transport in porous media," International Journal of Heat and Mass Transfer, vol. 46, no. 2, pp. 367-379, 2003.

[33] D. A. Nield and B. Adrian, Convection in Porous Media, Springer, Berlin, Germany, 2nd edition, 1999.

[34] A. K. Al-Hadhrami, L. Elliott, and D. B. Ingham, "Combined free and forced convection in vertical channels of porous media," Transport in Porous Media, vol. 49, no. 3, pp. 265-289, 2002.

[35] A. K. Al-Hadhrami, L. Elliott, and D. B. Ingham, "A new model for viscous dissipation in porous media across a range of permeability values," Transport in Porous Media, vol. 53, no. 1, pp. 117-122, 2003.

[36] E. R. G. Eckert and R. M. Drake, Analysis of Heat and Mass Transfer, McGraw-Hill, New York, 1977.

[37] D. A. Nield, A. V. Kuznetsov, and M. Xiong, "Thermally developing forced convection in a porous medium: parallel plate channel with walls at uniform temperature, with axial conduction and viscous dissipation effects," International Journal of Heat and Mass Transfer, vol. 46, no. 4, pp. 643-651, 2003.

[38] P. M. Jordan, A. Puri, and G. Boros, "On a new exact solution to Stokes' first problem for Maxwell fluids," International Journal of Non-Linear Mechanics, vol. 39, no. 8, pp. 1371-1377, 2004. 


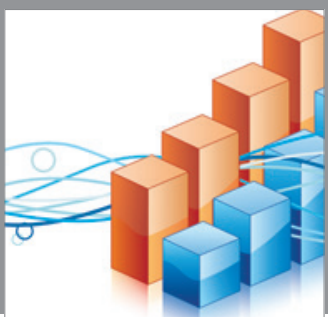

Advances in

Operations Research

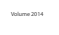

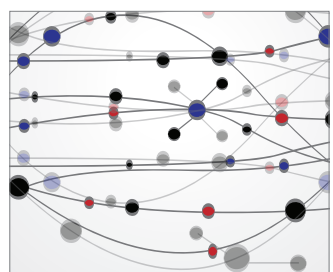

\section{The Scientific} World Journal
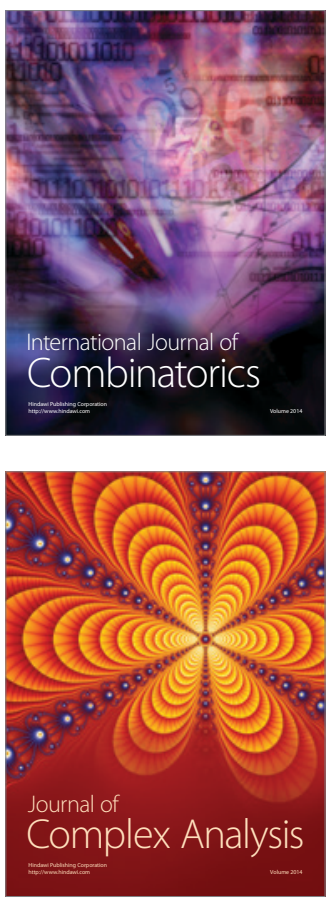

International Journal of

Mathematics and

Mathematical

Sciences
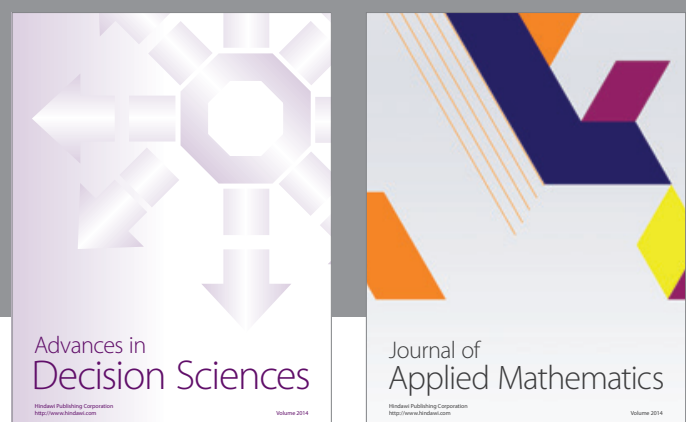

Journal of

Applied Mathematics
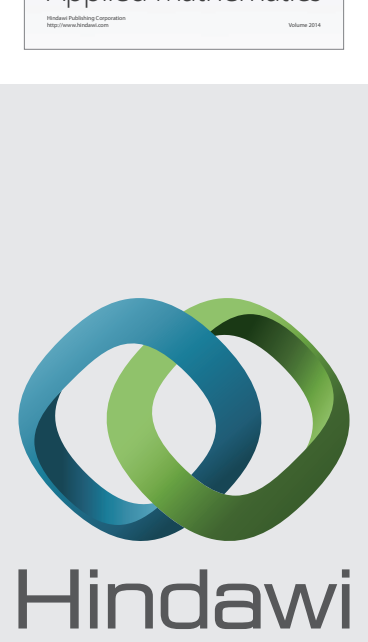

Submit your manuscripts at http://www.hindawi.com
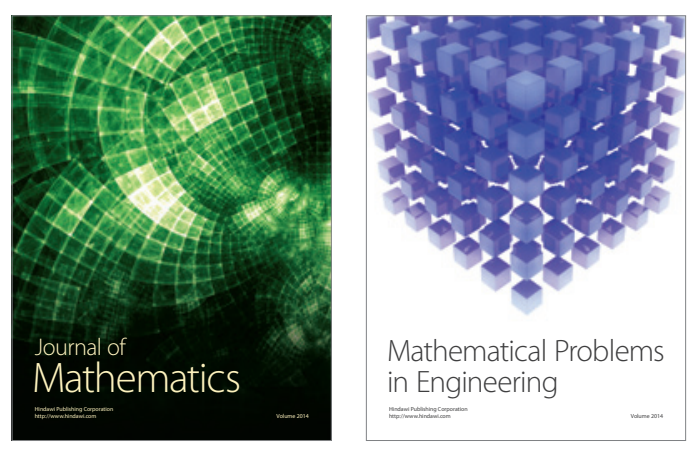

Mathematical Problems in Engineering
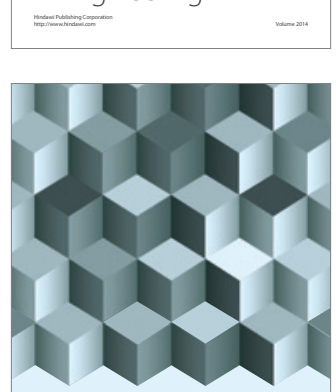

Journal of

Function Spaces
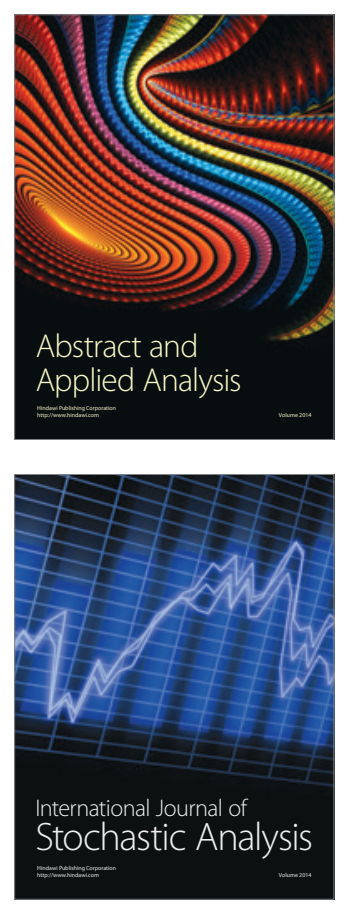

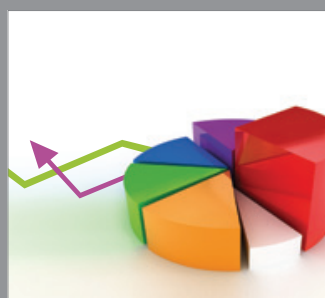

ournal of

Probability and Statistics

Promensencen
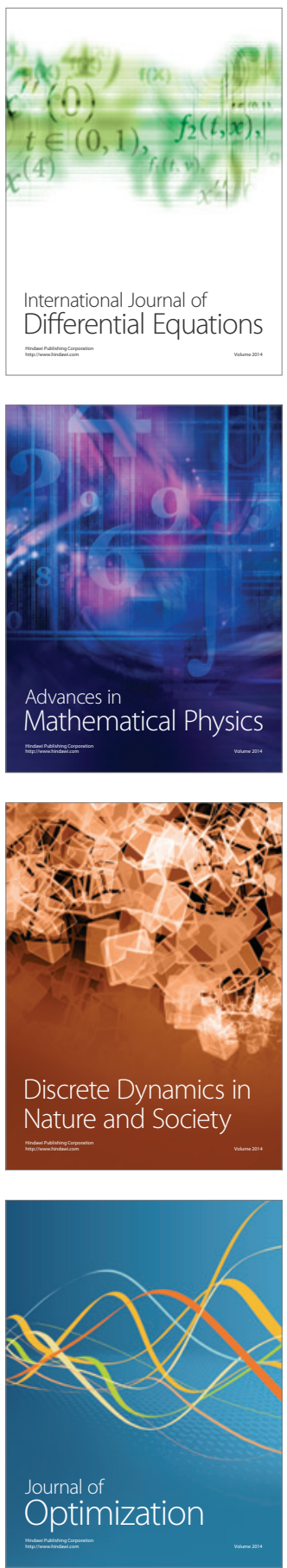